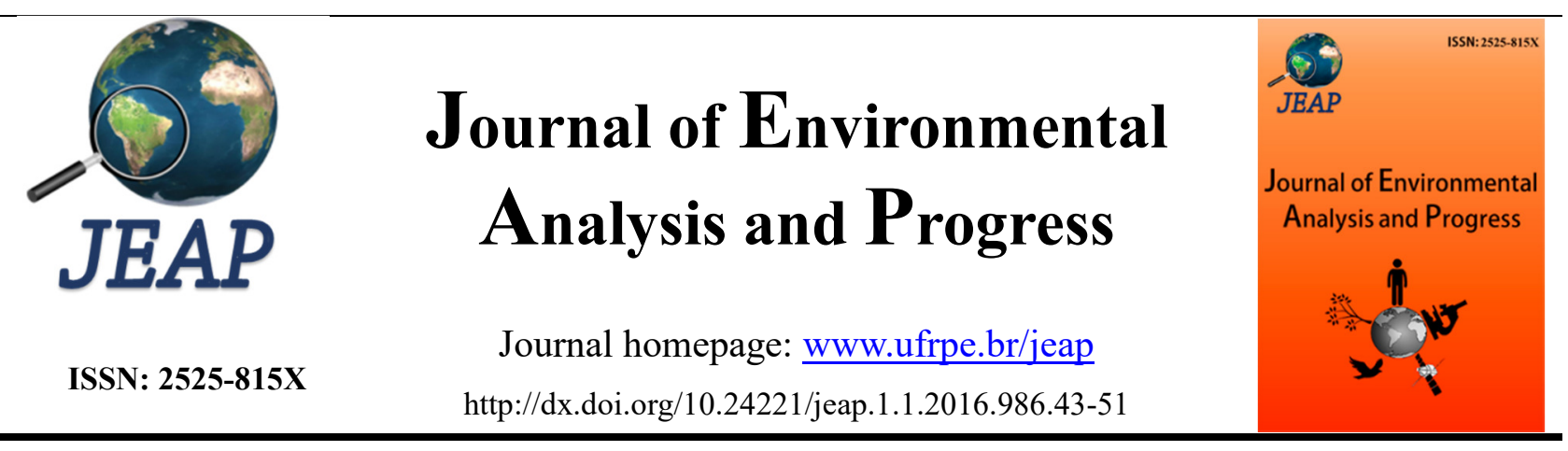

\title{
Floristic survey of the Caatinga in areas with different grazing intensities, Pernambuco, Northeast Brazil
}

\author{
Fernanda Meira Tavares ${ }^{1}$, Katharina Schulz ${ }^{2}$, Rita de Cássia Araújo Pereira ${ }^{3}$, Arne Cierjackss, \\ Jarcilene Silva de Almeida-Cortez ${ }^{1,5}$ \\ ${ }^{1}$ Universidade Federal Rural de Pernambuco-UFRPE, Programa de Pós-Graduação em Ecologia-PPGE. Rua Dom \\ Manoel de Medeiros, s/n, Dois Irmãos, Recife-PE, Brasil. CEP: 52171-900. \\ ${ }^{2}$ Technische Universität Berlin, Department of Ecology, Ecosystem Science/Plant Ecology, Rothenburgstraße 12, \\ Berlin. Germany, 12165. \\ ${ }^{3}$ Instituto Agronômico de Pernambuco-IPA, Herbário Dárdano de Andrade Lima, Av. General San Martin, 1371, \\ Bongi, Recife-PE, Brazil. CEP: 50761-000. \\ ${ }^{4}$ Universität Hamburg, Biodiversity of Useful Plants Working Group, Biocenter Klein Flottbek, Biodiversity of Useful \\ Plants, Ohnhorststraße 18, Hamburg, Germany. 22609. \\ ${ }^{5}$ Universidade Federal de Pernambuco-UFPE, Departamento de Botânica, Centro de Ciências Biológicas, Av. Professor \\ Nelson Chaves, 1235, Cidade Universitária, Recife-PE, Brazil. CEP: 50670-420. E-mail: jacortez@ufpe.br.
}

\begin{tabular}{|c|c|}
\hline A R T I C L E I N F O & A B S T RA C T \\
\hline $\begin{array}{l}\text { Received } 29 \text { Sep } 2016 \\
\text { Accepted } 10 \text { Oct } 2016 \\
\text { Published } 13 \text { Oct } 2016\end{array}$ & $\begin{array}{l}\text { A floristic survey was conducted in eighteen areas in the municipalities of Itacuruba } \\
\text { and Floresta, Pernambuco, northeast Brazil. The objective was to investigate if the } \\
\text { species richness of terrestrial plant species of the Caatinga is affected by grazing } \\
\text { intensity. Eighteen } 20 \times 20 \mathrm{~m}^{2} \text { plot were established in areas of low grazing intensity } \\
\text { (9), and areas with high grazing intensity (9). We recorded } 136 \text { species belonging to } \\
97 \text { genera and } 43 \text { families. The most species-rich families were Poaceae (14), } \\
\text { Fabaceae (13), and Asteraceae (11). The most species-rich genera were Aristida } \\
\text { (Poaceae), Sida (Malvaceae) and Ipomoea (Convolvulaceae). The number of species } \\
\text { in each study area (Itacuruba and Floresta) varied according to the distribution of the } \\
\text { precipitation, the soil types, and the actual land-use. Areas with a low grazing } \\
\text { pressure show a higher species richness of plant species than areas with a higher } \\
\text { grazing intensity. } \\
\text { Keywords: anthropic pressure, land-use history, plant species richness, semiarid } \\
\text { region. }\end{array}$ \\
\hline
\end{tabular}

\section{Introduction}

Brazil is the country with the largest area of tropical forests in the world. It also exhibits the highest biodiversity and with 43.902 species comprises the highest number of plant species of the world (Ribeiro \& Walter, 1998; Barbosa \& Peixoto, 2003; Forzza et al., 2012). Otherwise, the literature shows that in the tropical regions, rainforests have been more widely studied than dry forests, even though approximately $42 \%$ of tropical forests around the world are dry tropical ones (Murphy \& Lugo, 1986; Miles et al., 2006; Quesada et al., 2009; Espírito-Santo et al., 2009; Santos et al., 2011).
The largest tropical dry forest of South America can be found in the northeast of Brazil. It is called Caatinga and covers about $70 \%$ of the northeast with an area of approximately 844.453 $\mathrm{km}^{2}$ (MMA, 2014). The mean annual precipitation varies between 380 and $800 \mathrm{~mm}$ (Araújo et al., 2007). The Caatinga is characterized by shrubby and tree species, forming different assemblages of which many are practically unknown from an ecological point of view (Pereira et al., 2001). The vegetation of this region occurs to be noncontinuous due to the high spatial and temporal variability of rain events. In general, the vegetation is characterized by xerophytic species as deciduous trees and shrubs of short stature, which often 
exhibit thorns and spines and small leaves (Prado, 2003; Maracajá \& Benevides, 2006). Giullieti et al. (2002) state that the Caatinga exhibits a high biodiversity and shows a considerable number of endemic species: 596 woody species are already recorded of which 180 are endemic to the Caatinga. Including the herbaceous species, this number will be much higher (EMBRAPA, 2005). Herbaceous species are annual and strongly depend on the rainy season (Silva et al., 2012; MMA, 2014). According to Silva et al. (2009), 587 herbaceous species can be registered only for the semiarid area of Pernambuco.

Many tree and herb species, which can be found in the Caatinga, are already studied regarding their economic value (Lucena et al., 2006). The tree species are important for the supply of timber and are used in the pharmaceutical and cosmetics industry. The herbs play a very important ecologic and economic role as they are used for medical purposes, apiculture and as livestock fodder.

In contrast to studies carried out in other vegetation types of Brazil, only recently the flora of the Caatinga was studied regarding the effect of grazing pressure (Schulz et al., 2016). However, studies about the effect of grazing show that goat breeding is one of the main reasons for degradation in the arid areas of the world (Oba, 1998; Carmel \& Kadmon, 1999). Rook \& Tailowin (2003) affirm that grazing has an important impact on the dynamic of this vegetation type. The herbivory, mainly of domestic and wild ungulates, is known to influence vegetation (Huntly, 1991; Milchunas \& Lauenrith, 1993; Vavra et al., 2007) due to processes related to competitive abilities, which change density and frequency of the plants involved (Dias Filho et al., 2008).

The effect of herbivory on the vegetation may be both direct and indirect. The direct effects are caused by the consumption of plant biomass.
Immediately as a consequence of reduced leaf area and mass of leaves and roots, the rate of $\mathrm{CO}_{2}$ sequestration and the absorption of water and nutrients decrease. The indirect effects are a result of the change in the soil properties, the microclimate, nutrient cycles and competition between plants.

Santos et al. (2014) highlight the importance of carrying out more studies to understand the influence of climatic changes on Caatinga vegetation and ecosystem services to better manage the resources by implementing socioenvironmental policies, engaging the local population and providing subsidies for life wealth improvement. These are key aspects of a long-term recovery and conservation of the Brazilian dry tropical forest.

This study has the main goal to compare how the vegetation structure changes under different pressure of herbivory in areas with high and low grazing intensities at Caatinga.

\section{Material and Methods \\ Study area}

The floristic inventory was carried out on eighteen areas divided in nine areas with high and nine with low grazing intensity, located in the two municipalities of Floresta and Itacuruba. The grazing pressure was classified according to the farm owner information of the number of domestic animals (goats, sheep, cows, and donkeys) present in the area.

The study area is located in the mesoregion of São Francisco at Pernambuco, adjacent to the Itaparica Reservoir. The altitude asl. ranges between 300 and $1.050 \mathrm{~m}$. According to the classification of Köppen (1948), the study area belongs to the climate type BSs'h', with mean annual temperatures of $25,8^{\circ} \mathrm{C}$ and monthly temperature up to $33,9^{\circ} \mathrm{C}$ (Fig 1). The mean annual precipitation is $623 \mathrm{~mm}$ (Embrapa, 2001).

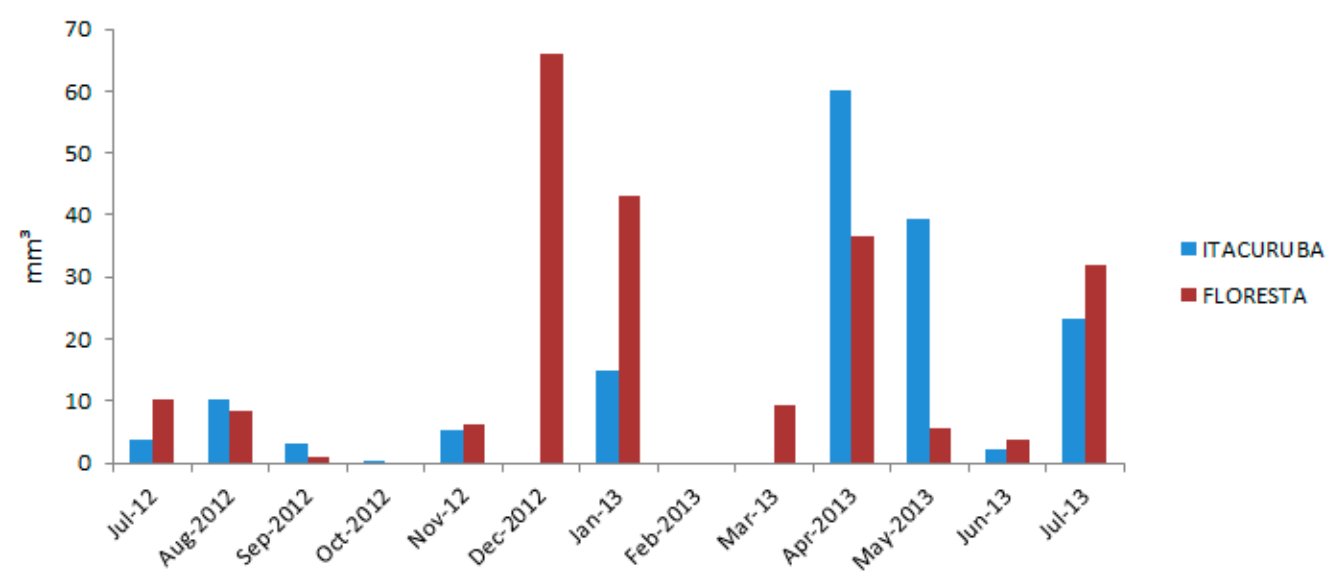

Figure 1. Monthly rainfall precipitations in 2012 and 2013. The months highlighted represent the period of sampling of herbaceous species in the study areas in the municipalities of Forest and Itacuruba. 
Inventory of woody species, bromeliads, cacti, and herbs

A permanent plot measuring $20 \mathrm{~m} \times 20 \mathrm{~m}$ was established in each of the eighteen areas. On each plot, plant diversity was assessed by carrying out vegetation relevés according to the methodology of Braun-Blanquet. Fertile branches, flowers, and leaf material were collected for plant identification. Plant identification was carried out in cooperation with the herbarium Dardano de Andrade of the Instituto Agronômico de Pernambuco (IPA), Recife, and followed Cronquist (1988) and APG III classification. The voucher material is deposited at the herbarium Dárdano de Andrade Lima (IPA) and Technische Universität Berlin. Some species did not have sufficient material to proceed the identification, probably caused by a huge drought in the years of the studies.

Table 1 . The list of species classified as woody species (W), bromeliads (B), cactus $(\mathrm{C})$ and herbs $(\mathrm{H})$ harvested at high (HP) and low pressure (LP) of livestock herbivory in areas with different land use at de Floresta e Itacuruba, Pernambuco.

\begin{tabular}{|c|c|c|c|c|}
\hline Family & Species & 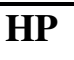 & $\mathbf{L P}$ & \\
\hline Alismataceae & Echinodorus lanceolatus Rataj & $\mathrm{X}$ & - & $\mathrm{H}$ \\
\hline \multirow[t]{4}{*}{ Amarantaceae } & Alternanhtera tenella Colla & - & $X$ & $\mathrm{H}$ \\
\hline & Amaranthus viridis $\mathrm{L}$. & - & $\mathrm{X}$ & $\mathrm{H}$ \\
\hline & Froelichia humboldtiana (Roem. \& Schult.) Seub. & - & $\mathrm{X}$ & $\mathrm{H}$ \\
\hline & Gomphrena demissa Mart. & $\mathrm{X}$ & $\mathrm{X}$ & $\mathrm{H}$ \\
\hline \multirow[t]{2}{*}{ Anacardiaceae } & Astronium urundeuva Engl. & - & $\mathrm{X}$ & $\mathrm{W}$ \\
\hline & Commiphora leptoploeos (Mart.) J. B. Gillett & - & $\mathrm{X}$ & $\mathrm{W}$ \\
\hline Apocynaceae & Aspidosperma pyrifolium Mart. & $\mathrm{X}$ & $\mathrm{X}$ & $\mathrm{W}$ \\
\hline \multirow{11}{*}{ Asteraceae } & Bidens pilosa $\mathrm{L}$. & - & $\mathrm{X}$ & $\mathrm{H}$ \\
\hline & Blainvillea acmella (L.) Philipson & - & $\mathrm{X}$ & $\mathrm{H}$ \\
\hline & Centratherum punctatum Caas. & $X$ & $X$ & $\mathrm{H}$ \\
\hline & Eclipta prostrata $(\mathrm{L}.) \mathrm{L}$. & $X$ & $X$ & $\mathrm{H}$ \\
\hline & Emilia forbergii Nicolson & $\mathrm{X}$ & - & $\mathrm{H}$ \\
\hline & Emilia sonchifolia (L.) DC. ex Wight & $X$ & - & $\mathrm{H}$ \\
\hline & Lagascea mollis Cav. & $\mathrm{X}$ & $\mathrm{X}$ & $\mathrm{H}$ \\
\hline & Pectis linifolia $\mathrm{L}$. & $\mathrm{X}$ & $\mathrm{X}$ & $\mathrm{H}$ \\
\hline & Pectis oligocephala (Gardner) Sch.Bip. & - & $\mathrm{X}$ & $\mathrm{H}$ \\
\hline & Solidago chilensis Mexer & - & $X$ & $\mathrm{H}$ \\
\hline & Tridax procumbens $\mathrm{L}$. & $\mathrm{X}$ & $\mathrm{X}$ & $\mathrm{H}$ \\
\hline \multirow[t]{3}{*}{ Boraginaceae } & Cordia leucocephala Moric & $\mathrm{X}$ & $\mathrm{X}$ & $\mathrm{W}$ \\
\hline & Heliotropium procumbens Mill. & - & $\mathrm{X}$ & $\mathrm{H} / \mathrm{W}$ \\
\hline & Heliotropium ternatum Vahl. & - & $X$ & $\mathrm{~W}$ \\
\hline \multirow[t]{3}{*}{ Bromeliaceae } & Bromelia laciniosa Mart. Ex. Schultes f. & $\mathrm{X}$ & - & $\mathrm{B}$ \\
\hline & Encholirium spectabile Martius ex Schultes \& Schultes f & $\mathrm{X}$ & $\mathrm{X}$ & $\mathrm{B}$ \\
\hline & Neoglaziovia variegata (Arruda) Mez. & $\mathrm{X}$ & $\mathrm{X}$ & $\mathrm{B}$ \\
\hline \multirow[t]{8}{*}{ Cactaceae } & Cereus jamacaru DC. & $\mathrm{X}$ & $\mathrm{X}$ & $\mathrm{C} / \mathrm{W}$ \\
\hline & Melocactus bahiensis (Britton \& Rose) Luetzelb.* & - & $\mathrm{X}$ & $\mathrm{C}$ \\
\hline & Melocactus zehntneride Britton \& Rose & - & $\mathrm{X}$ & $\mathrm{C}$ \\
\hline & Opuntia palmadora Britton \& Rose & - & $\mathrm{X}$ & $\mathrm{C}$ \\
\hline & Pilosocereus chrysostele (Vaupel) Byles \& Gdrowley & $\mathrm{X}$ & - & $\mathrm{C} / \mathrm{W}$ \\
\hline & Pilosocereus gounellei F.A.C.Weber & $\mathrm{X}$ & $\mathrm{X}$ & $\mathrm{C} / \mathrm{W}$ \\
\hline & Tacinga inamoena Backeb & $\mathrm{X}$ & $\mathrm{X}$ & $\mathrm{C}$ \\
\hline & Tacinga palmadora (Britton \& Rose) N.P.Taylor \& Stuppy & $\mathrm{X}$ & $\mathrm{X}$ & $\mathrm{C}$ \\
\hline \multirow[t]{2}{*}{ Capparaceae } & Cleome guianensis Aubl. & - & $\mathrm{X}$ & $\mathrm{H}$ \\
\hline & Cleome lanceolata (Mart. \& Zucc.) Iltis & - & $\mathrm{X}$ & $\mathrm{H}$ \\
\hline
\end{tabular}

Tavares, F. M.; Schulz, K.; Pereira, R. C. A.; Cierjacks, A.; Almeida-Cortez, J. S. 
Journal of Environmental Analysis and Progress V. 01 N. 01 (2016) 43-51

\begin{tabular}{|c|c|c|c|c|}
\hline Celastraceae & Maytenus rigida Mart. & $\mathrm{X}$ & - & $\mathrm{W}$ \\
\hline \multirow[t]{2}{*}{ Commelinaceae } & Commelina erecta $\mathrm{L}$. & - & $\mathrm{X}$ & $\mathrm{H}$ \\
\hline & Commelina obliqua Valh. & - & $\mathrm{X}$ & $\mathrm{H}$ \\
\hline \multirow[t]{8}{*}{ Convovulaceae } & Evolvulus frankenioides Moric. & $\mathrm{X}$ & $\mathrm{X}$ & $\mathrm{H}$ \\
\hline & Evolvulus glomeratus Nees \& C. Mart. & $\mathrm{X}$ & $\mathrm{X}$ & $\mathrm{H}$ \\
\hline & Ipomoea asarifolia Roem. \& Schult. & - & $\mathrm{X}$ & $\mathrm{H}$ \\
\hline & Ipomoea brasiliana Meisn & $\mathrm{X}$ & $\mathrm{X}$ & $\mathrm{H}$ \\
\hline & Ipomoea longeramosa Choisy & - & $\mathrm{X}$ & $\mathrm{H}$ \\
\hline & Ipomoea subrevoluta Choisy & $\mathrm{X}$ & - & $\mathrm{H}$ \\
\hline & Jacquemontia evolvuloides (Moric.) Meisn & $\mathrm{X}$ & $\mathrm{X}$ & $\mathrm{H}$ \\
\hline & Jacquemontia racemosa Meisn & $\mathrm{X}$ & - & $\mathrm{H}$ \\
\hline \multirow[t]{3}{*}{ Cyperaceae } & Cyperus odoratus L. & $\mathrm{X}$ & $\mathrm{X}$ & $\mathrm{H}$ \\
\hline & Cyperus surinamensis Rottb. & $\mathrm{X}$ & $\mathrm{X}$ & $\mathrm{H}$ \\
\hline & Lipocarpha micrantha (Vahl.) G. C. Tucker & $\mathrm{X}$ & $\mathrm{X}$ & $\mathrm{H}$ \\
\hline Gentianaceae & Schultesia guianensis (Aubl.) Malme & - & $\mathrm{X}$ & $\mathrm{H}$ \\
\hline \multirow[t]{8}{*}{ Euphorbiaceae } & Chamaesyce hirta (L.) Millsp. & $X$ & $\mathrm{X}$ & $\mathrm{H}$ \\
\hline & Chamaesyce thymifolia (L.) Millsp. & $\mathrm{X}$ & $\mathrm{X}$ & $\mathrm{H}$ \\
\hline & Cnidoscolus quercifolius Pohl & $X$ & $\mathrm{X}$ & W \\
\hline & Croton cordiifolius Baill. & - & $\mathrm{X}$ & $\mathrm{W}$ \\
\hline & Croton heliotropiifolius Kunth. & - & $\mathrm{X}$ & W \\
\hline & Croton hirtus $\mathrm{L}$. & - & $\mathrm{X}$ & $\mathrm{H}$ \\
\hline & Jatropha mollissima (Pohl) Baill. & $X$ & $\mathrm{X}$ & W \\
\hline & Jatropha ribifolia (Pohl) Baill. & - & $\mathrm{X}$ & $\mathrm{W}$ \\
\hline \multirow[t]{13}{*}{ Fabaceae } & Anadenanthera colubrina var. cebil (Griseb.) Altschul. & - & $\mathrm{X}$ & $\mathrm{W}$ \\
\hline & Bauhinia cheilantha (Bong.) Steud. & $\mathrm{X}$ & - & $\mathrm{W}$ \\
\hline & Caesalpinia ferrea C. Mart. & $\mathrm{X}$ & - & $\mathrm{W}$ \\
\hline & Caesalpinia microphylla G. Don. & $\mathrm{X}$ & - & $\mathrm{W}$ \\
\hline & Indigofera suffruticosa Mill. & $\mathrm{X}$ & $\mathrm{X}$ & $\mathrm{H} / \mathrm{W}$ \\
\hline & Mimosa tenuiflora (Willd.) Poir. & $\mathrm{X}$ & $\mathrm{X}$ & W \\
\hline & Neptunia plena (L.) Benth & $\mathrm{X}$ & $\mathrm{X}$ & $\mathrm{H}$ \\
\hline & Phyllanthus heteradenius Müll. Arg & $\mathrm{X}$ & - & $\mathrm{H}$ \\
\hline & Poincianella pyramidalis Tul. & $\mathrm{X}$ & $\mathrm{X}$ & $\mathrm{W}$ \\
\hline & Prosopis juliflora (Sw.) DC. & $\mathrm{X}$ & $\mathrm{X}$ & $\mathrm{W}$ \\
\hline & Senna obtusifolia (L.) H.S.Irwin \& Barneby & - & $\mathrm{X}$ & $\mathrm{H}$ \\
\hline & Stylosanthes scabra Vogel & $\mathrm{X}$ & - & $\mathrm{H} / \mathrm{W}$ \\
\hline & Zornia brasiliensis Vogel & $\mathrm{X}$ & - & $\mathrm{H}$ \\
\hline Hydroleaceae & Hydrolea spinosa $\mathrm{L}$. & - & $\mathrm{X}$ & $\mathrm{H}$ \\
\hline \multirow[t]{3}{*}{ Lamiaceae } & Hyptis suaveolens (L.) Poit & $\mathrm{X}$ & - & $\mathrm{H} / \mathrm{W}$ \\
\hline & Marsypianthes chamaedry (Vahl) Kuntze & - & $X$ & $\mathrm{H}$ \\
\hline & Ocimum campechianum Mill. & - & $\mathrm{X}$ & $\mathrm{H}$ \\
\hline Loganiaceae & Spigelia polystachya Klotzsch ex Prog. & - & $\mathrm{X}$ & $\mathrm{H}$ \\
\hline \multirow[t]{2}{*}{ Lytraceae } & Ammannia auriculata Willd & $\mathrm{X}$ & $\mathrm{X}$ & $\mathrm{H}$ \\
\hline & Cuphea campestres (Mart.) Koehne & - & $\mathrm{X}$ & $\mathrm{H} / \mathrm{W}$ \\
\hline \multirow[t]{3}{*}{ Malvaceae } & Herissantia tiubae (K. Schum.) Brit. & $\mathrm{X}$ & - & $\mathrm{W}$ \\
\hline & Pavonia cancellata (L.) Cav. & - & $\mathrm{X}$ & $\mathrm{H} / \mathrm{W}$ \\
\hline & Sida cordifolia $\mathrm{L}$. & $\mathrm{X}$ & $\mathrm{X}$ & $\mathrm{H} / \mathrm{W}$ \\
\hline
\end{tabular}


Journal of Environmental Analysis and Progress V. 01 N. 01 (2016) 43-51

Molluginaceae

Nyctaginaceae

Onagraceae

Phytolaccaceae

Poaceae

Polygalaceae

Portulacaceae

Plumbaginaceae

Rhamnaceae

Rubiaceae

Sapotaceae

Selaginellaceae

Schrophulariaceae

Solanaceae

Sterculiaceae

Sida galheirensis Ulbr.

Sida harleyi Krapov

Sida spinosa L.

Mollugo verticillata $\mathrm{L}$.

Boerhavia diffusa $\mathrm{L}$.

Ludwigia octovalvis (Jacq.) P.H. Raven

Microtea paniculata Moq

Anthephora hermaphrodita (L.) Kuntze

Aristida adscensionis L.

Aristida elliptica (Nees) Kunth

Aristida longifolia Trin.

Aristida megapotamica Spreng.

Aristida setifolia Kunth

Eragrostis amabilis (L.) Wight \& Arn

Eragrostis ciliaris (L.) R. Br.

Eragrostis pilosa (L.) P. Beauv

Melinis repens (Willd.) Zizka

Neesiochloa barbata (Nees) Pilger

Paspalum sp.1

Paspalum sp. 2

Tragus berteronianus Schult

Polygala brizoides A. St.-Hil. \& Moq.

Portulaca elatior Mart.

Portulaca oleracea $\mathrm{L}$.

Plumbago scandens L.

Crumenaria decumbens Mart.

Ziziphus joazeiro Mart.

Borreria scabiosoides Cham. \& Schltdl

Diodia apiculata (Willd. ex Roem. \& Schult.) K.Schum.

Diodia teres Walt.

Mitracarpus longicalyx E.B.Souza \& M.F.Sales

Richardia grandiflora (Cham. \& Schltdl.) Steud.

Staelia aurea K. Schum.

Sideroxylon obtusifolium (Roem. \& Schult.) T.D.Penn.

Selaginella convoluta (Arn.) Spring

Selaginella sellowii Hier

Angelonia campestris Nees \& Mart.

Angelonia gardneri Hook.

Scoparia ducis L.

Stemodia foliosa Benth.

Stemodia maritima $\mathrm{L}$.

Datura stramonium L.

Nicotiana glauca $\mathrm{R}$. Grah

Physalis angulata $\mathrm{L}$.

Physalis neesiana Sendtn.

Waltheria cf. indica $\mathrm{L}$.

Waltheria rotundifolia Schrank

\begin{tabular}{|c|c|c|}
\hline$X$ & $X$ & $\mathrm{H} / \mathrm{W}$ \\
\hline - & $X$ & $\mathrm{H} / \mathrm{W}$ \\
\hline- & $X$ & $\mathrm{H}$ \\
\hline$X$ & $X$ & $\mathrm{H}$ \\
\hline$X$ & $X$ & $\mathrm{H}$ \\
\hline- & $\mathrm{X}$ & $\mathrm{H}$ \\
\hline$X$ & $X$ & $\mathrm{H}$ \\
\hline$X$ & $X$ & $\mathrm{H}$ \\
\hline$X$ & $X$ & $\mathrm{H}$ \\
\hline$X$ & $X$ & $\mathrm{H}$ \\
\hline$X$ & $X$ & $\mathrm{H}$ \\
\hline$X$ & $X$ & $\mathrm{H}$ \\
\hline$X$ & $X$ & $\mathrm{H}$ \\
\hline$X$ & $X$ & $\mathrm{H}$ \\
\hline$X$ & $X$ & $\mathrm{H}$ \\
\hline$X$ & $X$ & $\mathrm{H}$ \\
\hline$X$ & $X$ & $\mathrm{H}$ \\
\hline$X$ & $X$ & $\mathrm{H}$ \\
\hline- & $X$ & $\mathrm{H}$ \\
\hline$X$ & - & $\mathrm{H}$ \\
\hline$X$ & $X$ & $\mathrm{H}$ \\
\hline$X$ & $X$ & $\mathrm{H}$ \\
\hline$X$ & $X$ & $\mathrm{H}$ \\
\hline- & $X$ & $\mathrm{H}$ \\
\hline- & $X$ & $\mathrm{~W}$ \\
\hline- & $X$ & $\mathrm{H} / \mathrm{W}$ \\
\hline- & $X$ & W \\
\hline$X$ & $X$ & $\mathrm{~W}$ \\
\hline$X$ & $X$ & $\mathrm{H} / \mathrm{W}$ \\
\hline$X$ & $X$ & $\mathrm{H} / \mathrm{W}$ \\
\hline- & $X$ & $\mathrm{H} / \mathrm{W}$ \\
\hline- & $X$ & $\mathrm{H}$ \\
\hline$X$ & $X$ & $\mathrm{H}$ \\
\hline$X$ & - & W \\
\hline$X$ & $X$ & $\mathrm{H}$ \\
\hline$X$ & - & $\mathrm{H}$ \\
\hline - & $X$ & $\mathrm{H} / \mathrm{W}$ \\
\hline- & $X$ & $\mathrm{H} / \mathrm{W}$ \\
\hline$X$ & - & $\mathrm{H}$ \\
\hline- & $X$ & W \\
\hline$X$ & - & $\mathrm{H}$ \\
\hline- & $X$ & $\mathrm{H} / \mathrm{W}$ \\
\hline- & $X$ & W \\
\hline- & $X$ & $\mathrm{H}$ \\
\hline- & $X$ & $\mathrm{H}$ \\
\hline$X$ & $X$ & $\mathrm{H}$ \\
\hline $\mathrm{X}$ & $\mathrm{X}$ & $\mathrm{H}$ \\
\hline
\end{tabular}


Journal of Environmental Analysis and Progress V. 01 N. 01 (2016) 43-51

\begin{tabular}{llccc} 
Tiliaceae & Corchorus hirtus L. & - & $\mathrm{X}$ & $\mathrm{H}$ \\
Turneraceae & Piriqueta cistoides (L.) Griseb. & $\mathrm{X}$ & $\mathrm{X}$ & $\mathrm{H}$ \\
& Piriqueta guianensis N.E.Br. & $\mathrm{X}$ & $\mathrm{X}$ & $\mathrm{H}$ \\
& Turnera pumilea Poir. & $\mathrm{X}$ & $\mathrm{X}$ & $\mathrm{H}$ \\
& Turnera subulata Sm. & $\mathrm{X}$ & $\mathrm{X}$ & $\mathrm{H}$ \\
Verbenaceae & Lantana camara L. & $\mathrm{X}$ & $\mathrm{X}$ & $\mathrm{W}$ \\
& Stachytarpheta sanguinea Mart. ex Schauer & - & $\mathrm{X}$ & $\mathrm{H}$ \\
Violaceae & Hybanthus arenarius Ule & - & $\mathrm{X}$ & $\mathrm{H}$ \\
& Hybanthus calciolaria (L.) Oken & $\mathrm{X}$ & - & $\mathrm{H}$ \\
Zygophilaceae & Tribulus terrestris L. & $\mathrm{X}$ & - & $\mathrm{H}$ \\
\hline
\end{tabular}

\section{Results}

The floristic inventory of woody species, cacti, bromeliads, and herbs registered 43 families, 97 genera, and 136 species in the studies areas at municipalities of Itacuruba and Floresta (Table 1). The most species-rich families were Poaceae (14), Fabaceae (13), Asteraceae (11), Euphorbiaceae (8) and Convolvulaceae (8). The most species-rich genera were: Aristida (Poaceae) with five species, followed by Sida (Malvaceae) and Ipomoea (Convolvulaceae) with four species each and Eragrostis (Poaceae) with three species.

Twenty-five tree species were recorded out of twelve families. Fifty-two herb species were recorded in the studies areas of Floresta, that represents $38,3 \%$ of the total species number collected during the survey. Cacti were represented by five generea and eight species, and bromeliads by three genera and three species: Bromelia laciniosa (a species endemic to the Caatinga); Encholirium spectabile and Neoglaziovia variegata. Two fern species of the Selaginellaceae family were harvested. (Table 1).

Fifty-five species were found in both municipalities, which represents $41,9 \%$ of all herbaceous species. Twenty-seven herbaceous species were found in the areas located at municipality of Itacuruba, which represents 19,9\%.

In the visited areas, twenty-three species were found in areas with high grazing pressure. Fifty-three species were found in the areas with a low grazing intensity. Sixty species were found in both grazing classes.

\section{Discussion}

Previously floristic survey was done by Souza et al. (2010) at the municipality of Floresta. The authors identified seventy-eight species, distributed within thirty-nine families. The most abundant families were Fabaceae (with thirteen genera and thirteen species), Euphorbiaceae (with six genera and eight species) and Asteraceae (with seven genera and seven species). The most abundant species were herbs with $43,6 \%$ of all recorded species, followed by trees $(17,95 \%)$, subshrubs and shrubs $(15,38 \%)$, vines $(5,12 \%)$, hemiparasites $(1,28 \%)$, and one rosette plant $(1,28 \%)$.

Similar results were gained during this study, where most species were herbs $(86 \%)$ and woody species represented $14 \%$.

Accordingly, the highest richness was observed in herbs species when comparing woody and non-woody species, corroborating previously studies done in different land use (Barbosa et al., 2003; Araújo et al., 2005; Rodal et al., 2005).

Silva et al. (2009) found ninety-five herbaceous species belonging to seventy-five genera and thirty-nine families on crystalline and sedimentary soils in Petrolândia, Pernambuco. The species of the families Poaceae, Euphorbiaceae, Asteraceae, Capparaceae, Convolvulaceae, Cyperaceae, Fabaceae, and Malvaceae were found in areas with sedimentary soils. The families Poaceae, Euphorbiaceae, Convolvulaceae, Portulacaceae, Bromeliaceae, Malvaceae, and Rubiaceae were found in areas with crystalline soils. Together they accounted for $49 \%$ of the total species number. These results are similar to the results found in our study. However, results differ for the families Fabaceae, Capparaceae, Portulacaceae, Bromeliaceae and Cyperaceae which in our study were present with two to three species. We may have distinguished species of the families Poaceae, Euphorbiaceae, Asteraceae, Capparaceae, Convolvulaceae, Cyperaceae, Fabaceae, Malvaceae as commonly occurring at the sedimentary soil region, from species of the families: Poaceae, Euphorbiaceae, Convolvulaceae, Portulacaceae, Bromeliaceae, Malvaceae, and Rubiaceae occurring at the crystalline soil region. Those species represent $49 \%$ of all species found by Silva et al. (2009). These results are very similar to our studies.

The floristic survey conducted by Silva et al. (2012) was carried out in an area of Caatinga, Crioulas, Ceará. The farmland involved is grazed by goats. Within a period of six months, thirty 
herbaceous species were found, belonging to fifteen families. Benevides et al. (2007), studying two areas, one was characterized by anthropogenic use, the second one was preserved to some extent. In the used area, they found thirty-two herbaceous species belonging to sixteen families. In the preserved area they found twenty-seven species belonging to twenty families. In contrast to the study conducted by Benevides et al. (2007), we found a higher number of species in the area with low grazing pressure.

Areas with low grazing intensity were only found in the municipality of Floresta. When we compare high and low herbivory pressure area, we found a higher species richness in low grazing intensity areas. One explanation could be the effects of soil degradation by the animal which may add to the pasture effects. Accordingly, Schulz et al. (2016) showed that grazing at high intensities significantly reduced soil carbon stocks of the upper soil horizons. Further, goats are the most common livestock in this region, and this animal has a generalist habit, eating different parts of the plant from different species, even those presenting chemical and/or mechanical defenses as spines.

\section{Conclusion}

The number of species in each study area (Itacuruba and Floresta) varied according to the distribution of the precipitation, the soil types, and the actual land-use. Areas with a low grazing pressure showed a higher species richness of plant species than areas with a higher grazing intensity. This implies that an intense grazing may leads to a decrease in species number, and thus directly changes the composition of the herbaceous species community.

\section{Acknowledgements}

This study was financed by the Federal Ministry of Education and Research of Germany (BMBF, 01LL0904A/E), the Ministry of Science, Technology and Innovation of Brazil (MCTI/CNPq, 490003/2012-5) and the Pernambuco Research Foundation (Facepe, APQ0842-2,05/12). Almeida-Cortez was supported by a fellowship from Brazilian CNPq (PQ 307422/2012-7).

\section{References}

ARAÚJO, E. L.; SILVA, K. A.; FERRAZ, E. M. N.; SAMPAIO, E. V. S. B.; SILVA, S. I. 2005. Diversidade de herbáceas em microhabitats rochoso, plano e ciliar em uma área de caatinga, Caruaru, PE, Brasil. Acta Botânica Brasílica. v.19, n.2, p.285-294.
ARAÚJO, E. L.; CASTRO, C. C.; ALBUQUERQUE, U. P. 2007. Dynamics of Brazilian Caatinga - A Review Concerning the Plants, Environment and People. Functional Ecosystems and Communities, v.1, n.1, p.15-28.

BARBOSA, M. R.V.; PEIXOTO, A. L. 2003. Coleções botânicas brasileiras: situação atual e perspectivas. In: PEIXOTO, et al. (ed.). Coleções biológicas de apoio ao inventário, uso sustentável e conservação da biodiversidade. Rio de Janeiro: Instituto de Pesquisas Jardim Botânico. pp.113125.

BARBOSA D. C. A.; BARBOSA M. C. A.; LIMA L. C. M.; 2003. Fenologia de espécies lenhosas da Caatinga. In: LEAL, I. R.; TABARELLI, M. (eds) Ecologia e Conservação da Caatinga. Editora Universitária UFPE, Recife, pp 657-693.

BENEVIDES, D. S.; MARACAJÁ, P. B.; SIZENANDO FILHO, F. A.; GUERRA, A. M. N. DE M.; PEREIRA, T. F. C. 2007. Estudo da flora herbácea da Caatinga no Município de Caraúbas no Estado do Rio Grande do Norte. Revista Verde. v.2 n.1, p.33-34.

CARMEL, Y.; KADMON, R. 1999. Effects of Grazing and Topography on Long Term Vegetation Changes in a Mediterranean Ecosystem in Israel. Plant Ecology, v.145, p.239-250.

COSTA, R. C.; ARAÚJO, F.S.; LIMA-VERDE, L.W. 2007. Flora and life-form spectrum in an area of deciduous thorn woodland (Caatinga) in northeastern Brazil. Journal of Arid Environments, v.68, p.237-247.

DIAS FILHO, M. B.; FERREIRA, J. N. 2008. Influência do pastejo na biodiversidade do ecossistema da pastagem. In: PEREIRA, O. G.; OBEID, J. A.; FONSECA, D. M. DA; NASCIMENTO JÚNIOR, D. DO. (Ed.). Simpósio sobre manejo estratégico da pastagem. Viçosa: Universidade Federal de Viçosa, pp. 47-74.

$\begin{array}{lrr}\text { EMPRESA BRASILEIRA DE } & \text { PESQUISA } \\ \text { AGROPECUÁRIA-EMBRAPA. } & 2001 .\end{array}$ Diagnóstico Ambiental do Município de Floresta, Pernambuco. Rio de Janeiro-RJ: EMBRAPA. (Circular Técnica, ISSN1517-5146). Disponível em:

http://www.cnps.embrapa.br/solosbr/pdfs/circulart ecnica10diagambfloresta pdf. Acesso em: 20 de janeiro de 2014. 
ESPÍRITO-SANTO M. M.; SEVILHA A. C.; ANAYA F. C.; BARBOSA R.; FERNANDES G. W.; SANCHEZ-AZOFEIFA G. A.; SCARIOT A.; NORONHA S. E.; SAMPAIO C. A. 2009 Sustainability of tropical dry forests: two case studies in southeastern and central Brazil. For. Ecol. Manag., v.258, p.922-930.

FORZZA, R. C.; LEITMAN, P. M.; COSTA, A. F.; CARVALHO JR., A. A.; PEIXOTO, A. L.; WALTER, B. M. T.; BICUDO, C.; ZAPPI, D.; COSTA, D. P.; LLERAS, E.; MARTINELLI, G.; LIMA, H. C.; PRADO, J.; STEHMANN, J. R.; BAUMGRATZ, J. F. A.; PIRANI, J. R.; SYLVESTRE, L.; MAIA, L. C.; LOHMANN, L. G.; QUEIROZ, L. P.; SILVEIRA, M.; COELHO, M. N.; MAMEDE, M. C.; BASTOS, M. N. C.; MORIM, M. P.; BARBOSA, M.; MENEZES, M.; HOPKINS, M.; SECCO, R.; CAVALCANTI, T. B.; SOUZA, V.C. 2012. Introdução. In: Lista de Espécies da Flora do Brasil. Jardim Botânico do Rio de Janeiro. Available: http://floradobrasil.jbrj. gov.br/2012/. Access: 08/04/2014.

GIULIETTI, A. M.; HARLEY, R. M.; QUEIROZ, L. P.; BARBOSA, M. R. V.; BOCAGE NETA, A. L.; FIGUEIREDO, M. A. Espécies endêmicas da Caatinga. In: SAMPAIO, E. V. S. B.; GIULIETTI, A. M.; VIRGÍNIO, J.; GAMARRAS-ROJAS, C. F. L. 2002. (orgs). Vegetação e flora da Caatinga, APNE/CNIP. pp. 103-119.

HUNTLY, N. 1991. Herbivores and the dynamics of communities and ecosystems. Annu. Rev. Eco. Syst., v.22, p.477-503.

KÖPPEN, W. 1948. Climatologia: con un estudio de los climas de la tierra. Fondo de Cultura Econômica. México.

LUCENA, R. F. P.; ARAUJO, E. L; ALBUQUERQUE, P. 2007. Does the local availability of woody Caatinga plants (Northeastern Brazil) explain their use value? Economic Botany, v.61, p.347-361.

MARACAJÁ, P. B.; BENEVIDES, D. S. 2006. Estudo da Flora Herbácea da Caatinga no Município de Caraúbas no Estado do Rio Grande do Norte. Revista de Biologia e Ciências da Terra, v.6, p.165-175.

MILCHUNAS, D. G.; LAUENROTH, W. K. 1993. Quantitative Effects of Grazing on Vegetation and Soils Over a Global Range of Environments. Ecological Monographs, v.63, n.4, p.328-366.
MILES, L.; NEWTON, A. C.; FRIES, R. S.; RAVILIOUS, C.; MAY, I.; BLYTH, S.; KAPOS, V.; GORDON, J. E. 2006. A global overview of the conservation status of tropical dry forests. J Biogeogr., v.33, p.491-505.

MMA-Ministério do Meio Ambiente. 2002. Available: $\quad$ http://www.mma.gov.b/sitio/ index.php?ido=conteudo.montaeidEstrutura=203. Access: 25 ago. 2013.

MURPHY, P. G.; LUGO, A. E. 1986. Ecology of tropical dry forest. Annual Review of Ecology and Systematics, v.17, p.67-88.

PEREIRA, I. M.; ANDRADE, L. A.; COSTA, J. R. M. DIAS, J. M. 2001. Análise da regeneração natural em um remanescente de caatinga sob diferentes níveis de perturbação, no agreste paraibano. Acta Botanica Brasilica, v.15, n.3, p.413-426.

PRADO, D. 2003. As caatingas da América do sul. In: LEAL, I. R.; TABARELI, M.; SILVA, J. M. C. (Eds.). Ecologia e conservação da caatinga. Editora Universitária, Universidade Federal de Pernambuco, Recife, pp. 3-73.

QUESADA, M.; SANCHEZ-AZOFEIFA, G. A.; ALVAREZ-ANORVE, M. 2009. Succession and management of tropical dry forests in the Americas: review and new perspectives. For. Ecol. Manag., v.258, p.1014-1024.

RIBEIRO, J. F.; WALTER, B. M. T. 1998. Fitofisionomias do Bioma Cerrado. pp.89-166. In: SANO, S. M.; ALMEIDA, S. P. (Eds.). Cerrado: ambiente e flora. Planaltina, EMBRAPA.

RODAL, M. J. N.; LINS E SILVA, A. C. B.; PESSOA, L. M.; CAVALCANTI, A. D. C. 2005. Vegetação e flora fanerogâmica da área de Betânia, Pernambuco. pp. 91-119. In: ARAÚJO, F. S.; RODAL, M. J. N.; BARBOSA, M. R. V. (orgs). Análise das variações da biodiversidade do bioma caatinga: suporte a estratégias regionais de conservação. Brasília, Ministério do Meio Ambiente.

ROOK, A. J.; TALLOWIN, J. R. B. Grazing and pasture management for biodiversity benefit. Animal Research, v.52, p.181-189, 2003.

SANTOS, J. C.; LEAL, I. R.; ALMEIDACORTEZ, J. S.; FERNANDES, G. W.; TABARELLI, M. 2011. Caatinga: the scientific 
negligence experienced by a dry tropical forest. Trop. Conserv. Sci., v.4, p.276-286.

SANTOS, M. G.; OLIVEIRA, M. T.; FIGUEIREDO, K. V.; FALCÃO, H.; ARRUDA, E. C. P.; ALMEIDA-CORTEZ, J. S.; SAMPAIO, E. V. S. B.; OMETTO, J. P.; MENEZES, R. S. C.; OLIVEIRA, A. F. M.; POMPELLI, M. F.; ANTONINO, A. C. D. 2014. Caatinga, the Brazilian dry tropical forest: can it tolerate climate changes? Theoretical and Experimental Plant Physiology, v.26, n.1, p.83-99

SILVA, K. A.; ARAUJO, E. L.; FERRAZ, E. M. N. 2009. Estudo florístico do componente herbáceo e relação com solos em áreas de caatinga do embasamento cristalino e bacia sedimentar, Petrolândia-PE. Acta Botânica Brasília, v.23, n.1, p.100-110.
SILVA, B. L. R.; TAVARES, F. M.; ALMEIDACORTEZ, J S. 2012. Composição florística do componente herbáceo de uma área de caatinga Fazenda Tamanduá, Paraíba, Brasil. Revista de Geografia, v.29, p.54-64.

SCHULZ, K.; VOIGT, K.; BEUSCH, C.; ALMEIDA-CORTEZ, J. S.; KOWARIK, I.; WALZ, A.; CIERJACKS, A. 2016. Grazing deteriorates the soil carbon stocks of Caatinga forest ecosystems in Brazil. Forest Ecology and Management, v.367, p.62-70.

VAVRA, M.; PARKS, C. G.; WISDOM, M. J. 2007. Biodiversity, exotic plant species, and herbivory: The good, the bad, and the ungulate. Forest Ecology and Management, v.246, n.1, p.6672. 